\title{
A Randomized, Double-Blind, Placebo-Controlled Study of the Safety and Efficacy of Olanzapine for the Prevention of Chemotherapy-Induced Nausea and Vomiting in Patients Receiving Moderately Emetogenic Chemotherapy: Results of the Korean South West Oncology Group (KSWOG) Study
}

\author{
So-Yeon Jeon, $\mathrm{MD}^{1,2}$ \\ Hye Sook Han, MD, PhD ${ }^{3}$ \\ Woo Kyun Bae, MD, PhD ${ }^{4}$ \\ Moo-Rim Park, MD, PhD \\ Hyeok Shim, MD ${ }^{5}$ \\ Sang-Cheol Lee, MD, $\mathrm{PhD}^{6}$ \\ Se-II Go, MD \\ Hwan Jung Yun, MD \\ Yong-Jin Im, MS 9 \\ Eun-Kee Song, MD, PhD',2
}

\begin{abstract}
${ }^{1}$ Department of Internal Medicine, Chonbuk National University Medical School, Jeonju, ${ }^{2}$ Research Institute of Clinical Medicine of Chonbuk National University, Biomedical Research Institute of Chonbuk National University Hospital, Jeonju, ${ }^{3}$ Department of Internal Medicine, Chungbuk National University College of Medicine, Cheongiu, ${ }^{4}$ Department of Internal Medicine, Chonnam National University Hwasun Hospital, Hwasun, ${ }^{5}$ Department of Internal Medicine, Wonkwang University School of Medicine, Iksan, ${ }^{6}$ Department of Internal Medicine, Soonchunhyang University Hospital, Cheonan, ${ }^{7}$ Department of Internal Medicine, Gyeongsang National University Hospital, Gyeongsang National University School of Medicine, Jinju, ${ }^{8}$ Department of Internal Medicine, Chungnam National University College of Medicine, Daejeon, ${ }^{9}$ Center for Clinical Pharmacology and Biomedical Research Institute, Chonbuk National University Hospital, Jeonju, Korea
\end{abstract}

\section{Purpose}

Data on the efficacy of olanzapine in patients receiving moderately emetogenic chemotherapy (MEC) are limited. This study aimed to evaluate and compare the efficacy of olanzapine versus placebo in controlling nausea and vomiting in patients receiving MEC.

\section{Materials and Methods}

We conducted a randomized, double-blind, placebo-controlled study to determine whether olanzapine can reduce the frequency of chemotherapy-induced nausea and vomiting (CINV) and improve the quality of life (QOL) in patients receiving palonosetron and dexamethasone as prophylaxis for MEC-induced nausea and vomiting. The primary end point was complete response for the acute phase (0-24 hours after chemotherapy). The secondary end points were complete response for the delayed (24-120 hours) and overall phase (0-120 hours), proportion of significant nausea (visual analogue scale $\geq 25 \mathrm{~mm}$ ), use of rescue medications, and effect on QOL.

\section{Results}

Fifty-six patients were randomized to the olanzapine $(n=29)$ and placebo $(n=27)$ groups. Complete response rates were not significantly different between the olanzapine and placebo groups in the acute $(96.5 \%$ vs. $88.0 \%, p=0.326)$, delayed $(69.0 \%$ vs. $48.0 \%$, $p=0.118)$, and overall phases $(69.0 \%$ vs. $48.0 \%, p=0.118)$. However, the percentage of patients with significant nausea $(17.2 \%$ vs. $44.0 \%, p=0.032)$ and the use of rescue medications $(0.03 \pm 0.19$ vs. $1.88 \pm 2.88, p=0.002)$ were lower in the olanzapine group than in the placebo. Furthermore, the olanzapine group demonstrated better QOL $(p=0.015)$.

\section{Conclusion}

Olanzapine combined with palonosetron and dexamethasone significantly improved QOL and vomiting control among previously untreated patients receiving MEC, although the efficacy was limited to the reduction of the frequency of CINV.

Key words

Olanzapine, Antiemetics, Nausea, Vomiting
Correspondence: Eun-Kee Song, MD, PhD Department of Internal Medicine, Chonbuk National University Medical School, 20, Geonjiro, Deokjin-gu, Jeonju 54907, Korea Tel: 82-63-250-1245

Fax: 82-63-254-1609

E-mail: eksong@jbnu.ac.kr

Received December 6, 2017 Accepted February 26, 2018 Published Online February 27, 2018 


\section{Introduction}

Chemotherapy-induced nausea and vomiting (CINV) is the most common and debilitating side effect of cytotoxic chemotherapy. Up to $85 \%$ of treated cancer patients can be affected by CINV resulting in the deterioration of their quality of life (QOL) and compliance to anti-cancer treatments, which adversely affects the treatment outcome. Furthermore, CINV causes metabolic imbalances, worsening nutritional status, and degradation of body function and impairs daily life function $[1,2]$. In patients receiving chemotherapy, the frequency and degree of nausea and vomiting are affected by various factors such as the types and dosages of anticancer drugs, treatment schedules and routes of administration, and the individual characteristics (for example, sex, age, previous chemotherapy treatment, and alcohol consumption) $[3,4]$. The use of 5-hydroxytryptamine type 3 (5-HT3) receptor antagonists, dexamethasone, and neurokinin-1 (NK1) receptor antagonists has significantly improved the control of CINV, and international guidelines recommend the combinations of these agents to prevent CINV in patients receiving moderately or highly emetogenic chemotherapy (HEC) [5-7].

Olanzapine is widely used as an antipsychotic drug for patients with schizophrenia and intractable depression. Olanzapine blocks multiple neurotransmitters with their receptors: dopamine at D1, D2, D3, and D4 receptors; serotonin at 5-HT2a, 5-HT2c, 5-HT3, and 5-HT6 receptors; catecholamines at alpha- 1 adrenergic receptors; acetylcholine at muscarinic receptors; and histamine at $\mathrm{H} 1$ receptors $[8,9]$. In a recent phase 3 trial, olanzapine significantly prevented nausea and improved the complete response rate, compared with placebo, among previously untreated patients who were receiving HEC [10]. In another single-institution phase 3 trial, olanzapine combined with dexamethasone and palonosetron, an HT3-receptor blocker, was effective in controlling acute and delayed nausea and vomiting in patients receiving HEC [11]. Based on these results, an olanzapinecontaining regimen has been recommended as one of the standard prophylactic treatment in patients receiving HEC.

However, only few studies have evaluated the efficacy of olanzapine in patients receiving moderately emetogenic chemotherapy (MEC). In a small randomized trial, the addition of olanzapine to standard therapy can reduce the frequency of CINV and improve the QOL of patients receiving HEC or MEC [12]. However, less than 10 patients in each group received MEC in this trial. Furthermore, palonosetron and dexamethasone combination therapy is equally recommended irrespective of containing olanzapine in patients receiving MEC. In addition, the dosage of olanzapine and dexamethasone was arbitrarily recommended without pro- per evaluation.

The purpose of this study was to evaluate the efficacy of olanzapine versus that of a placebo, in controlling nausea and vomiting in patients receiving MEC. The objective was to determine the additive effect of olanzapine to palonosetron and dexamethasone combination therapy among previously untreated patients receiving MEC.

\section{Materials and Methods}

\section{Patient selection}

Patients aged 19 years or older with malignant disease who had not received previous chemotherapy within 6 months before enrollment were eligible if (1) they were scheduled to receive MEC (either carboplatin, cyclophosphamide $\leq 1,500$ $\mathrm{mg} / \mathrm{m}^{2}$; daunorubicin, doxorubicin $\leq 60 \mathrm{mg} / \mathrm{m}^{2}$; epirubicin $\leq 90 \mathrm{mg} / \mathrm{m}^{2}$; irinotecan, oxaliplatin, melphalan, or methotrexate $\geq 250 \mathrm{mg} / \mathrm{m}^{2}$ ) and (2) had a European Cooperative Oncology Group (ECOG) performance status of 0,1 , or 2. Additional eligibility criteria were (1) an aspartate or alanine aminotransferase level that was no more than 3 times the upper limit of the normal range, creatinine clearance of 50 $\mathrm{mL} / \mathrm{min}$ or more, an absolute neutrophil count of at least $1,500 / \mathrm{mm}^{3}$, and a platelet count of at least $100,000 / \mathrm{mm}^{3}$; (2) no nausea or vomiting in the 24 hours before enrollment; (3) no severe cognitive compromise; no known history of central nervous system disease; (4) no treatment with another antipsychotic agent such as risperidone, quetiapine, clozapine, phenothiazine, or butyrophenone within 30 days before enrollment or plans for such treatment during the study period; (5) no concurrent abdominal radiotherapy; (6) no chronic alcoholism; (7) no known hypersensitivity to olanzapine, palonosetron, and dexamethasone; (8) no treatment with any 5-HT3 receptor antagonist, benzamide, domperidone, cannabinoid, NK1 receptor antagonist, and benzodiazepine within 4 weeks before enrollment; (9) no known cardiac arrhythmia, uncontrolled congestive heart failure, or acute myocardial infarction within the previous 6 months; (10) no history of uncontrolled diabetes mellitus; and (11) negative result of pregnancy test in women of childbearing age.

\section{Study design}

All patients eligible for the study were randomized to either the olanzapine or placebo group with palonosetron and dexamethasone regimen according to a computer-generated random assignment schedule created by an independ- 
ent statistician not involved with the study. Patients were further stratified according to sex and the participating centers. The patients and the medical professionals who cared for them were unaware of the assigned study regimen. The patients were assessed for only one chemotherapy cycle.

\section{Treatment regimen}

On the day of chemotherapy (day 1), all patients received an antiemetic regimen consisting of $12 \mathrm{mg}$ dexamethasone and $0.25 \mathrm{mg}$ palonosetron both administered intravenously 30 minutes prior to chemotherapy administration. In addition, the patients received $10 \mathrm{mg}$ olanzapine administered orally every day or a matching placebo on days 1-4. Patients were permitted to take rescue therapy of the investigator's choice for nausea and vomiting based on clinical circumstances excluding NK1 receptor antagonist, olanzapine, and readministration of palonosetron.

\section{Study visits and assessment procedures}

All the patient's demographic and medical data were recorded during the screening period. Patients were asked to complete daily records of episodes of vomiting or nausea and the use of rescue therapy from day 1-6. Patients were also asked to record every day the number and time of any emetic events and maximum nausea experienced, which was rated using a visual analogue scale (VAS) ranging from 0 ("no nausea at all") to 10 ("nausea as bad as it can be") [13]. Adverse events were graded according to the terminology and grading categories defined in the National Cancer Institute's Common Terminology Criteria for Adverse Events, ver. 4.03. In addition, patients completed the Korean version of the Functional Living Index-Emesis (FLI-E) questionnaire, which is a self-administered and validated questionnaire, on day 6 to assess the influence of CINV on QOL. The FLI-E questionnaire contains 18 questions (nine for nausea and nine for vomiting) that specifically address the effects of nausea and vomiting on physical activities, social and emotional functions, and the ability to enjoy meals [14]. Scores range from 1 to 7 for each domain, with a higher score indicating a more favorable and better ability to maintain daily activities.

\section{Outcomes}

The primary end point in this study was complete response (CR), defined as no emetic episodes and no use of rescue medication in the acute ( $0-24$ hours post-chemotherapy) phase. The secondary end points were $C R$ in the delayed (24-120 hours post-chemotherapy) and overall (0-120 hours post-chemotherapy) phases, no vomiting and no significant nausea (VAS $<25 \mathrm{~mm}$ ) in the overall phase, use of rescue medications, and QOL of patients as assessed using the FLI-E questionnaire.

\section{Statistical analysis}

Based on $80 \%$ power in detecting an achievement from $70 \%$ to $97 \%$ with a 0.05 level of significance between the placebo and olanzapine groups $(\alpha=0.05,1-\beta=0.80), 26$ patients were required per group. Chi-square test was used to compare complete responses, no vomiting and no significant nausea in the overall phase. Wilcoxon rank-sum test was used for the comparison of use of rescue medication, QOL of patients as assessed using the FLI-E questionnaire. The percentage of patients with an average domain item score of

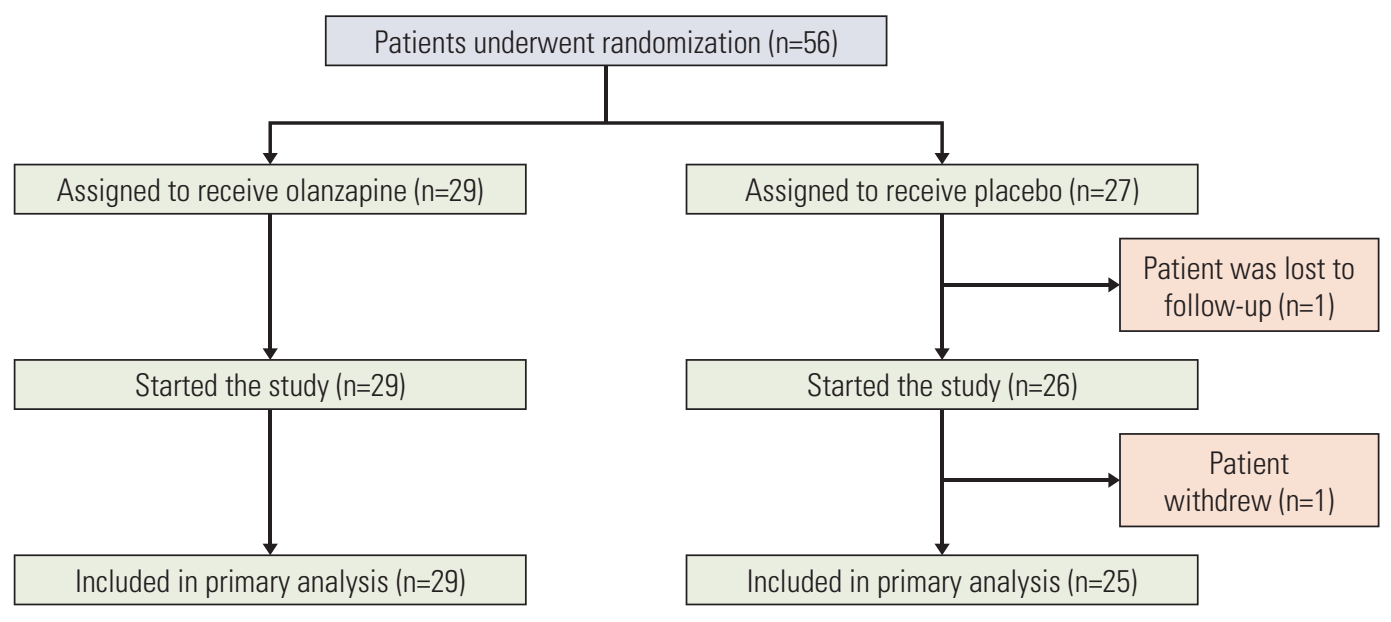

Fig. 1. Patients who underwent randomization, started the study, and were included in the primary analysis. 
Table 1. Baseline demographic and clinical characteristics of the study patients

\begin{tabular}{|c|c|c|}
\hline Characteristic & Olanzapine $(n=29)$ & Placebo $(n=25)$ \\
\hline \multicolumn{3}{|l|}{ Sex } \\
\hline Male & $23(79)$ & $22(88)$ \\
\hline Female & $6(21)$ & $3(12)$ \\
\hline \multicolumn{3}{|l|}{ Age (yr) } \\
\hline Median & 60 & 63 \\
\hline Range & $30-76$ & $42-79$ \\
\hline \multicolumn{3}{|l|}{ ECOG performance status } \\
\hline 0 & $6(21)$ & $3(12)$ \\
\hline 1 & $23(79)$ & $22(88)$ \\
\hline \multicolumn{3}{|l|}{ Chemotherapy regimen } \\
\hline Oxaliplatin containing & $16(55)$ & $14(56)$ \\
\hline Irinotecan containing & $11(38)$ & $9(36)$ \\
\hline Carboplatin containing & $2(7)$ & $2(8)$ \\
\hline \multicolumn{3}{|l|}{ Purpose of chemotherapy } \\
\hline Palliative & $16(55)$ & $18(72)$ \\
\hline Adjuvant & $13(45)$ & $6(24)$ \\
\hline Neoadjuvant & 0 & $1(4)$ \\
\hline \multicolumn{3}{|l|}{ Primary site of disease } \\
\hline Colorectal & $18(62)$ & $13(52)$ \\
\hline Stomach & $8(28)$ & $7(28)$ \\
\hline Lung & $2(7)$ & $2(8)$ \\
\hline Other & $1(3)$ & $3(12)$ \\
\hline \multicolumn{3}{|c|}{ Previous alcohol consumption } \\
\hline Yes & $13(45)$ & $5(20)$ \\
\hline No & $16(55)$ & $20(80)$ \\
\hline
\end{tabular}

Values are presented as number $(\%)$. ECOG, European Cooperative Oncology Group.

over 6 on the seven-point scale in the FLI-E questionnaire, which represent no effect of CINV on daily life activities, was calculated and compared between two groups.

\section{Ethical statement}

All patients gave written informed consent, and the study was approved by the institutional review board at each participating center.

\section{Results}

\section{Study patients}

Fig. 1 shows the distribution and randomization of the study patients. A total of 56 patients at seven academic institutions in the southwestern part of Korea were randomly assigned to the olanzapine $(n=29)$ and placebo $(n=27)$ groups between August 2015 and December 2016. However, of the 27 patients in the placebo group, one patient was lost to follow-up and another patient withdrew; thus, a total of 54 patients were included in the analysis.

The demographic and clinical characteristics of the 56 patients are presented in Table 1. No significant differences in sex, age, chemotherapy regimen administered, ECOG performance status, or primary site of disease was noted between the olanzapine and placebo groups. The majority of patients in each group were men, and the majority also received chemotherapy consisting of oxaliplatin or irinotecan plus fluoropyrimidine. The distribution of institutions was balanced between the study groups.

\section{Efficacy}

The CR for the acute, delayed, and overall phases is shown in Table 2. One patient in the olanzapine group who did not have a CR in the acute phase did not require rescue medication, but three patients in the placebo group required rescue medication and received additional antiemetics such as 
Table 2. Complete response according to study group

\begin{tabular}{|c|c|c|c|c|}
\hline Variable & Olanzapine ( $\mathrm{n}=29$ ) & Placebo $(n=25)$ & Total $(n=54)$ & p-value \\
\hline \multicolumn{5}{|c|}{ 0-24 Hours after chemotherapy } \\
\hline No & $1(3.5)$ & $3(12.0)$ & $4(7.4)$ & 0.326 \\
\hline Yes & $28(96.5)$ & $22(88.0)$ & $50(92.6)$ & \\
\hline \multicolumn{5}{|c|}{ 24-120 Hours after chemotherapy } \\
\hline No & $9(31.0)$ & $13(52.0)$ & $22(40.7)$ & 0.118 \\
\hline Yes & $20(69.0)$ & $12(48.0)$ & $32(59.3)$ & \\
\hline \multicolumn{5}{|c|}{ 0-120 Hours after chemotherapy } \\
\hline No & $9(31.0)$ & $13(52.0)$ & $22(40.7)$ & 0.118 \\
\hline Yes & $20(69.0)$ & $12(48.0)$ & $32(59.3)$ & \\
\hline
\end{tabular}

Values are presented as number $(\%)$.

Table 3. Other secondary end points analyzed according to the study group

\begin{tabular}{lcccc} 
Variable & Olanzapine $(\mathbf{n = 2 9})$ & Placebo $(\mathbf{n}=\mathbf{2 5})$ & Total $(\mathbf{n}=54)$ & p-value \\
Vomiting in the overall phase & & & & \\
$\quad$ No & $20(69.0)$ & $14(56.0)$ & $34(63.0)$ & 0.325 \\
$\quad$ Yes & $9(31.0)$ & $11(44.0)$ & $20(37.0)$ & \\
Significant vomiting in the overall phase & $24(82.8)$ & $14(56.0)$ & $38(70.4)$ & 0.032 \\
$\quad$ No & $5(17.2)$ & $11(44.0)$ & $16(29.6)$ & \\
$\quad$ Yes & $0.03 \pm 0.19$ & $1.88 \pm 2.88$ & & 0.002 \\
Frequency of rescue medication & $2.55 \pm 13.74$ & $19.44 \pm 28.23$ & & 0.004 \\
\hline Time to the use of rescue medication $(\mathbf{h r})$ &
\end{tabular}

Values are presented as number $(\%)$ or mean \pm standard deviation.

Table 4. Proportion of patients reporting "no impact of CINV on daily life"

\begin{tabular}{|c|c|c|c|}
\hline Variable & Olanzapine $(\mathrm{n}=29)$ & Placebo $(n=25)$ & p-value \\
\hline \multicolumn{4}{|l|}{ FLI-E total score } \\
\hline Mean \pm SD & $119.12+15.49$ & $107.81 \pm 22.27$ & 0.009 \\
\hline Median (min-max) & $125.98(64.97-125.98)$ & $120.22(40.50-125.98)$ & \\
\hline \multicolumn{4}{|l|}{ Nausea domain } \\
\hline Mean \pm SD & $58.48 \pm 9.95$ & $50.56 \pm 13.64$ & 0.004 \\
\hline Median (min-max) & $62.99(17.52-62.99)$ & $57.23(17.04-62.99)$ & \\
\hline \multicolumn{4}{|l|}{ Vomiting domain } \\
\hline Mean \pm SD & $60.64 \pm 7.06$ & $57.25 \pm 10.68$ & 0.078 \\
\hline Median (min-max) & $62.99(28.14-62.99)$ & $62.93(23.46-62.99)$ & \\
\hline \multicolumn{4}{|c|}{ Impact on daily life, $n(\%)$} \\
\hline No & $26(89.7)$ & $15(60.0)$ & 0.015 \\
\hline Yes & $3(10.3)$ & $10(40.0)$ & \\
\hline
\end{tabular}

CINV, chemotherapy-induced nausea and vomiting; FLI-E, the Functional Living Index-Emesis; SD, standard deviation. 
metoclopramide or 5-HT3 receptor antagonist for 2-8 times. Nine patients in the olanzapine group and thirteen patients in the placebo group did not have a CR in the delayed phase. The proportion of patients with a CR was not significantly different between the two groups in all three assessment periods. The $\mathrm{CR}$ rates during the acute, delayed, and overall phases were $96.5 \%$ vs. $88.0 \%$ ( $p=0.326$ ), $69.0 \%$ vs. $48.0 \%$ ( $\mathrm{p}=0.118)$, and $69.0 \%$ vs. $48.0 \%(\mathrm{p}=0.118)$, respectively (Table 2$)$.

A total of $20(69.0 \%)$ and $14(56.0 \%)$ patients in the olanzapine and placebo group, respectively, developed no vomiting in the overall phase. Meanwhile, the proportion of patients with significant nausea in the overall phase was significantly lower in the olanzapine group than in the placebo group ( 5 patients, $17.2 \%$ vs. 11 patients, $44.0 \%$; $\mathrm{p}=0.032$ ). The number of patients who required rescue medication and time to the administration of rescue medication in the olanzapine group were also lower to the placebo group (Table 3).

In terms of FLI-E scores for QOL, CINV did not affect the daily activities in 26 patients $(89.7 \%)$ in the olanzapine group and in 15 patients $(60.0 \%)$ in the placebo group (Table 4$)$. The FLI-E scores were significantly higher in olanzapine group compared to placebo $(\mathrm{p}=0.015)$.

\section{Adverse events}

The overall incidence, frequency, and intensity of adverse events were comparable between the two treatment groups. No grade 3 or 4 treatment-related adverse event occurred in both groups. The most common adverse event in the olanzapine group was somnolence. Four patients out of the 29 patients $(13.8 \%)$ experienced grade $1(n=3)$ or $2(n=1)$ somnolence. The other adverse events in the olanzapine group were grade 1 hypertriglyceridemia $(n=3)$, hyperglycemia $(n=1)$, and fatigue $(n=1)$. The most common adverse event in the placebo group was grade $1(n=1)$ and grade $2(n=2)$ fatigue. The other adverse events were grade 1 hypertriglyceridemia $(\mathrm{n}=2)$ and asthenia $(\mathrm{n}=1)$. No patient discontinued the study because of undesired adverse events.

\section{Discussion}

Over the past few decades, the development of new antiemetics remarkably improved the control of CINV. Typical antiemetic drugs include 5-HT3 receptor antagonists, NK1 receptor antagonists, and dexamethasone, and a new combination of these antiemetics like Netupitant and palonosetron (NEPA) has been developed. Although it was not originally developed as an antiemetic, the use of olanzapine for the control of CINV has gradually increased.
Recently, the American Society of Clinical Oncology guideline recommends a four-drug combination regimen comprising an NK1 receptor antagonist, a 5-HT3 receptor antagonist, dexamethasone, and olanzapine as prophylaxis for HECinduced nausea and vomiting; meanwhile, the National Comprehensive Cancer Network (NCCN) guideline recommends a three-drug combination regimen of palonosetron, dexamethasone, and olanzapine in both HEC- and MECinduced nausea and vomiting $[15,16]$. The only difference between these two regimens for HEC- and MEC-induced nausea and vomiting is the dosage of olanzapine $(10 \mathrm{mg}$ for 4 days in HEC regimen vs. $10 \mathrm{mg}$ for 3 days in MEC regimen). In addition, olanzapine is the drug of choice for breakthrough treatment as category 1 in the NCCN guideline [15].

The effect of a three-drug regimen that includes olanzapine has been proven in several phase 2 and 3 trials of various settings. However, to the best of our knowledge, data from a prospective study on the effect of olanzapine in patients receiving only MEC are yet to be available. Chiu et al. [17] conducted a literature search to identify randomized controlled trials that compared olanzapine to other standard antiemetics for either prevention of or rescue from CINV. They identified 13 eligible trials, with 10 trials in the preventative setting and three trials in the breakthrough setting. In the preventative setting, six studies included patients receiving only HEC, four studies included patients with a combination of HEC and MEC in various proportions, while no studies included patients receiving only MEC.

The first clinical study to report the effects of olanzapine was published in 2005 by Navari et al. [18]. In this study, 10 patients receiving HEC and 20 patients receiving MEC were enrolled, and a $100 \% \mathrm{CR}$ rate for acute phase was reported using three-drug regimens in both groups. Tan et al. [19] reported the effect of olanzapine-containing regimen in a cohort with both HEC and MEC combined; of them, approximately $50 \%$ patients received MEC. In this study, the CR rate in the acute phase was not significantly different in both HEC and MEC groups, but the significant level of nausea and vomiting in the delayed and overall phases was substantially improved in both groups. Mizukami et al. [12] conducted a randomized, double-blind, placebo-controlled trial to determine whether olanzapine can reduce the frequency of CINV and improve patient's QOL during chemotherapy. In this study, 44 patients receiving HEC and MEC were included, and the total control rate and improvement in QOL were significantly higher in the olanzapine group than in the control group. However, only 15 patients were receiving MEC in this study.

The primary end point of the current study was to compare the CR rate for the acute phase. At the time of the study plan, a difference of $27 \%$ (97\% vs. $70 \%$ ) between the two groups was expected, but the actual results were not statisti- 
cally significant at $96.5 \%$ for the olanzapine group and $88.0 \%$ for the placebo group. The secondary end point-the CR rate for the delayed and overall phases-was also insignificant between the two groups. Although these outcomes were not significantly different, a clear trend favoring the olanzapine group was observed. In previous clinical studies evaluating the efficacy of antiemetics, researchers used various parameters including the $\mathrm{CR}$ rate, the degrees of significant nausea, the necessity of taking rescue medication, and the time until administration of rescue medication. These parameters were included as secondary end points of the present study, and we were able to confirm that the degree of significant nausea and the necessity of taking rescue medication were significantly improved in the olanzapine group. Furthermore, the effects of chemotherapy on QOL, which was evaluated using the FLI-E questionnaire, was found to be significantly better in the olanzapine group than in the placebo group.

Olanzapine is a drug originally developed as an antipsychotic drug. Unlike other antiemetic agents, it blocks multiple receptors associated with emetic pathways. Therefore, additional caution is needed when it is used with other drugs such as metoclopramide and haloperidol, which can be used in cancer patients, because excessive dopamine inhibition increases the risk of extrapyramidal symptoms. However, olanzapine has been reported to be safe when used intermittently for preventive purposes after every cycle of chemotherapy or for breakthrough treatment. Because of the risk of central nervous system depression, extra care should be taken when using olanzapine in old, debilitated, or frail patients. The safety of olanzapine has been often evaluated through the M.D. Anderson symptom inventory. Fatigue, drowsiness, and disturbed sleep were the most commonly reported adverse events. However, these adverse events were infrequent and manageable. All patients in our study did not develop any clinically significant adverse event.

This study has several limitations. First, the number of patients is relatively small, although the design was a ran- domized, double-blinded, placebo-controlled study. Other limitations are that the effect of more than two cycles of chemotherapy had not been evaluated, and several factors associated with CINV, such as age and amount of alcohol consumption, were not included for the stratification. Therefore, a larger scale phase 3 study is necessary to confirm the effect of olanzapine in patients receiving MEC.

In some countries including Korea, olanzapine has not yet been approved as an antiemetic agent. This is because clinical trials using olanzapine have not been performed in these countries and various antiemetics are already available. Another reason is the misconception for antipsychotic drugs. Internationally, olanzapine is already among the recommended antiemetics, and it was shown to be more effective than other antiemetics in some patients. Furthermore, it has an advantage of being cost-effective compared to new highpriced antiemetics. Based on the results of this study, olanzapine should be considered more actively in patients receiving MEC.

Olanzapine in addition to palonosetron and dexamethasone could not significantly improve the $\mathrm{CR}$ rate for acute, delayed, and overall phase among previously untreated patients receiving MEC. However, a clear benefit of olanzapine was observed in the management of vomiting and QOL, and it can be recommended as an option for CINV prevention in Korean patients receiving MEC.

\section{Conflicts of Interest}

The authors thank all of the researchers and the participating heath centers including CJ Health Care for the kind donation of study drugs.

\section{Acknowledgments}

This paper was funded by the Biomedical Research Institute of Chonbuk National University Hospital.

\section{References}

1. Ingle RJ, Burish TG, Wallston KA. Conditionability of cancer chemotherapy patients. Oncol Nurs Forum. 1984;11:97-102.

2. Richardson JL, Marks G, Levine A. The influence of symptoms of disease and side effects of treatment on compliance with cancer therapy. J Clin Oncol. 1988;6:1746-52.

3. Herrstedt J. Antiemetics: an update and the MASCC guidelines applied in clinical practice. Nat Clin Pract Oncol. 2008;5:32-43.

4. Hesketh PJ, Grunberg SM, Herrstedt J, de Wit R, Gralla RJ,
Carides AD, et al. Combined data from two phase III trials of the NK1 antagonist aprepitant plus a 5HT 3 antagonist and a corticosteroid for prevention of chemotherapy-induced nausea and vomiting: effect of gender on treatment response. Support Care Cancer. 2006;14:354-60.

5. Hesketh PJ, Bohlke K, Lyman GH, Basch E, Chesney M, ClarkSnow RA, et al. Antiemetics: American Society of Clinical Oncology focused guideline update. J Clin Oncol. 2016;34: 381-6. 
6. Herrstedt J, Roila F, Warr D, Celio L, Navari RM, Hesketh PJ, et al. 2016 Updated MASCC / ESMO consensus recommendations: prevention of nausea and vomiting following high emetic risk chemotherapy. Support Care Cancer. 2017;25:27788.

7. Jordan K, Gralla R, Jahn F, Molassiotis A. International antiemetic guidelines on chemotherapy induced nausea and vomiting (CINV): content and implementation in daily routine practice. Eur J Pharmacol. 2014;722:197-202.

8. Geling O, Eichler HG. Should 5-hydroxytryptamine-3 receptor antagonists be administered beyond 24 hours after chemotherapy to prevent delayed emesis? Systematic re-evaluation of clinical evidence and drug cost implications. J Clin Oncol. 2005;23:1289-94.

9. Bymaster FP, Falcone JF, Bauzon D, Kennedy JS, Schenck K, DeLapp NW, et al. Potent antagonism of 5-HT(3) and 5-HT(6) receptors by olanzapine. Eur J Pharmacol. 2001;430:341-9.

10. Navari RM, Qin R, Ruddy KJ, Liu H, Powell SF, Bajaj M, et al. Olanzapine for the prevention of chemotherapy-induced nausea and vomiting. N Engl J Med. 2016;375:134-42.

11. Navari RM, Gray SE, Kerr AC. Olanzapine versus aprepitant for the prevention of chemotherapy-induced nausea and vomiting: a randomized phase III trial. J Support Oncol. 2011;9:18895.

12. Mizukami N, Yamauchi M, Koike K, Watanabe A, Ichihara K, Masumori N, et al. Olanzapine for the prevention of chemotherapy-induced nausea and vomiting in patients receiving highly or moderately emetogenic chemotherapy: a randomized, double-blind, placebo-controlled study. J Pain Symptom Manage. 2014;47:542-50.
13. Borjeson S, Hursti TJ, Peterson C, Fredikson M, Furst CJ, Avall-Lundqvist $\mathrm{E}$, et al. Similarities and differences in assessing nausea on a verbal category scale and a visual analogue scale. Cancer Nurs. 1997;20:260-6.

14. Martin AR, Pearson JD, Cai B, Elmer M, Horgan K, Lindley C. Assessing the impact of chemotherapy-induced nausea and vomiting on patients' daily lives: a modified version of the Functional Living Index-Emesis (FLIE) with 5-day recall. Support Care Cancer. 2003;11:522-7.

15. Hesketh PJ, Kris MG, Basch E, Bohlke K, Barbour SY, ClarkSnow RA, et al. Antiemetics: American Society of Clinical Oncology clinical practice guideline update. J Clin Oncol. 2017;35:3240-61.

16. Berger MJ, Ettinger DS, Aston J, Barbour S, Bergsbaken J, Bierman PJ, et al. NCCN guidelines insights: antiemesis, version 2.2017. J Natl Compr Canc Netw. 2017;15:883-93.

17. Chiu L, Chow R, Popovic M, Navari RM, Shumway NM, Chiu $\mathrm{N}$, et al. Efficacy of olanzapine for the prophylaxis and rescue of chemotherapy-induced nausea and vomiting (CINV): a systematic review and meta-analysis. Support Care Cancer. 2016;24:2381-92.

18. Navari RM, Einhorn LH, Passik SD, Loehrer PJ Sr, Johnson C, Mayer ML, et al. A phase II trial of olanzapine for the prevention of chemotherapy-induced nausea and vomiting: a Hoosier Oncology Group study. Support Care Cancer. 2005;13:529-34.

19. Tan L, Liu J, Liu X, Chen J, Yan Z, Yang H, et al. Clinical research of olanzapine for prevention of chemotherapyinduced nausea and vomiting. J Exp Clin Cancer Res. 2009; 28:131. 